\title{
Type B thymoma: Is prognosis predicted only by World Health Organization classification?
}

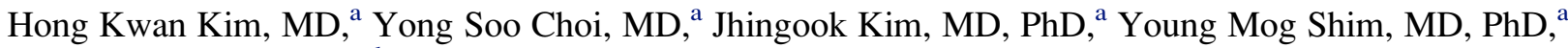 \\ Joungho Han, MD, PhD, ${ }^{\mathrm{b}}$ and Kwhanmien Kim, $\mathrm{MD}, \mathrm{PhD}^{\mathrm{a}}$
}

Objective: The prognostic relevance of subtypes within type B thymomas is controversial. The objective of this study was to evaluate the utility of World Health Organization (WHO) classification in patients with type B thymoma.

\begin{abstract}
Methods: This was a retrospective review of 100 patients who underwent thymectomy for WHO type B thymoma. Recurrence patterns and survival were compared among subtypes.
\end{abstract}

Results: There were 22 type B1 tumors, 43 type B2 tumors, and 35 type B3 tumors. Incomplete resection occurred in 5 patients with type B1 thymoma, 8 with type B2 thymoma, and 8 with type B3 thymoma $(P=.87)$. Of the 79 patients with complete resection, tumor recurrence occurred in $1(5.9 \%)$ patient with type B1 thymoma, $2(5.7 \%)$ with type B2 thymoma, and $2(7.4 \%)$ with type B3 thymoma, and all of these patients had Masaoka stage III disease. Disease-free survival at 5 years was $93 \%, 85 \%$, and $82 \%$ in type B1, B2, and B3, respectively (B1 vs B2; $P=.79$; B2 vs B3; $P=0.6$ ). Disease-free survival at 5 years was $94 \%, 100 \%, 61 \%$, and $50 \%$ in Masaoka stages I, II, III, and IV, respectively (I vs II; $P=.26$; II vs III; $P=.028$; III vs IV; $P=.002$ ).

Conclusions: Tumor recurrence was significantly associated with advanced Masaoka stage regardless of the WHO subtype of type B thymomas. Given the heterogeneity of WHO type B thymomas, Masaoka stage should always be considered when predicting prognosis and planning adjuvant treatment for patients with type B thymomas. (J Thorac Cardiovasc Surg 2010;139:1431-5)

Supplemental material is available online.

The World Health Organization (WHO) histologic types of thymomas are based on morphologic features ${ }^{1}$ and closely correlate with the Masaoka staging system, reflecting the extent and invasiveness of thymomas. ${ }^{2-7}$ However, when "biologically aggressive" type B2 or B3 thymomas are completely resected at an "early" Masaoka stage, it is difficult to determine the need for adjuvant treatment in such patients. It is still unclear whether predicting prognosis or planning adjuvant treatment can be based only on the WHO classification regardless of the Masaoka staging system. In particular, the prognostic relevance of subtypes within type B thymomas remains controversial, inasmuch as few studies have investigated the outcome and prognosis

From the Departments of Thoracic and Cardiovascular Surgery ${ }^{\mathrm{a}}$ and Pathology, Samsung Medical Center, Sungkyunkwan University School of Medicine, Seoul, Korea.

Disclosures: None.

Received for publication May 12, 2009; revisions received Sept 10, 2009; accepted for publication Oct 8, 2009; available ahead of print Dec 28, 2009

Address for reprints: Kwhanmien Kim, MD, Professor, Department of Thoracic and Cardiovascular Surgery, Samsung Medical Center, 50 Ilwon-dong, Gangnam-gu,

Seoul, 135-710, Korea (E-mail: kmkim0070@skku.edu).

$0022-5223 / \$ 36.00$

Copyright (C) 2010 by The American Association for Thoracic Surgery

doi:10.1016/j.jtcvs.2009.10.024 of patients with thymoma, specifically focusing on the WHO type B histology.

We hypothesized that the Masaoka staging system would reflect the disease status of patients with thymoma and thus predict the prognosis better than the WHO classification. The objectives of this study were (1) to compare the recurrence patterns and long-term survival of type B thymoma subtypes based on the Masaoka staging system as well as the WHO classification and (2) to evaluate the utility of the WHO classification in patients with type B thymomas.

\section{PATIENTS AND METHODS \\ Patients}

Between December 1994 and February 2008, 265 consecutive patients underwent thymectomy for thymic epithelial tumors at our institution. Of these, 100 patients had WHO type B thymomas and were included in the study population. Their medical records were retrospectively reviewed to compare the clinical characteristics, recurrence pattern, and long-term survival among B1, B2, and B3 thymoma subtypes. The study was reviewed and approved by the Institutional Review Board of Samsung Medical Center.

All patients had extended thymectomy, which was defined as the resection of the entire thymus and mediastinal fat tissue between both phrenic nerves. A median sternotomy was done in all patients except in cases in which a posterolateral thoracotomy was also needed so that an en bloc extrapleural pneumonectomy could be performed for unilateral pleural dissemination. Resection was defined as incomplete when microscopic (R1) or macroscopic (R2) residual tumor was present at the end of the surgical procedure. Mostly, patients with WHO type B2 or B3 thymomas or at advanced Masaoka stage III or IVa disease received adjuvant treatment, including radiation therapy, chemotherapy, or concurrent chemoradiation. 


\author{
Abbreviations and Acronyms \\ DFS = disease-free survival \\ OS $=$ overall survival \\ WHO $=$ World Health Organization
}

\begin{abstract}
Histology and Staging
With the use of hematoxylin-eosin stained sections derived from paraffin-embedded blocks, histologic diagnosis was re-evaluated on all specimens by a pathologist who was blinded to clinical information. Type B thymomas were subclassified according to the WHO classification as follows: type B1 thymomas are predominantly cortical, B2 thymomas are cortical, and B3 thymomas are well-differentiated thymic carcinomas. When thymomas contained more than one subtype (eg, combined B1 +B2 thymomas), they were categorized into the predominant subtype to facilitate analysis. Tumor stage was determined following the scheme suggested by Masaoka and associates. ${ }^{8}$ Stage I disease indicates macroscopically completely encapsulated thymomas with no microscopically determined capsular invasion; stage II indicates macroscopic invasion into the surrounding fatty tissue or mediastinal pleura, or microscopic invasion into the capsule; stage III indicates macroscopic invasion into neighboring organs (ie, pericardium, great vessels, lung); stage IVa indicates pleural or pericardial dissemination; and stage $\mathrm{IVb}$ indicates lymphogenous or hematogenous metastasis.
\end{abstract}

\section{Follow-up and Statistical Analysis}

Patients were regularly evaluated by computed tomography scans every 3 months for the first 2 years after the operation and then every 6 months thereafter. Student $t$ tests and the $\chi^{2}$ tests or Fisher's exact tests were used to compare the continuous and categorical variables, respectively. All deaths were considered as events, regardless of whether or not they were thymoma-related. Overall survival (OS) was defined as time from the date of thymectomy to death from any cause. Disease-free survival (DFS) was defined as time from the date of thymectomy to recurrence or death. OS and DFS were calculated by the Kaplan-Meier method. OS and DFS among subtypes were compared by the log-rank test. To determine which factors were significantly associated with survival, we performed a multivariate analysis using the Cox proportional hazards model.

\section{RESULTS}

\section{Clinical and Histopathologic Findings}

There were 52 men and 48 women, with a mean age of $50.8 \pm 12.9$ years, ranging from 23 to 79 years. Twentytwo $(22 \%)$ patients had type B1 thymomas, $43(43 \%)$ had type B2 thymomas, and $35(35 \%)$ had type B3 thymomas. Thirteen $(13 \%)$ patients had myasthenia gravis. The site of extrathymic invasion was lung in 15 patients, pericardium in 8 , superior vena cava in 3 , and innominate vein in 3 . The correlation between WHO subtype and Masaoka stage is summarized in Table 1. Although type B1 thymomas tended to correlate with early Masaoka stages, as compared with type B2 or B3 thymomas, the correlation was not statistically significant $(P=.7)$.

\section{Treatment Modalities and Complete Resection}

The treatment modalities performed in our series are summarized in Table E1 according to WHO histologic type.
Two (7\%) patients received neoadjuvant chemotherapy followed by extrapleural pneumonectomy owing to the possibility of stage IVa disease. Extrapleural pneumonectomy $(\mathrm{n}=3)$ and pneumonectomy with partial pleurectomy $(\mathrm{n}=2)$ was performed in 5 patients, and complete resection was achieved in 2 of those patients. Postoperative adjuvant therapy was given to $74(74 \%)$ patients. Sixty-seven $(67 \%)$ patients received adjuvant radiotherapy. There was no significant difference in the frequency of adjuvant treatment among the 3 subtypes $(P=.3)$. Complete tumor resection (R0) in types B1, B2, and B3 thymomas was achieved in $77 \%, 81 \%$, and $77 \%$, respectively. In 14 patients with stage IVa disease, multiple disseminated pleural nodules were unexpectedly detected at surgery, whereas there was focal microscopic involvement of the resection margin in 7 patients with stage III disease. Although the incidence of incomplete resection was not different among the 3 subtypes $(P=.87)$, all the tumors that were incompletely resected were either Masaoka stage III or IVa (Table 2).

\section{Recurrence and Survival}

No in-hospital mortality was noted during the postoperative period. The median follow-up time was 49 months, ranging from 5 to 159 months. No significant difference was found in the follow-up duration among the 3 subtypes $(P=$ .73). During follow-up, 6 patients died. The cause of death included radiation pneumonitis in 2 patients, cardiac toxicity owing to doxorubicin (Adriamycin) in 1, aplastic anemia in 1 , pneumonia resulting from myasthenic crisis in 1, and thymoma-related death in 1 . OS was $96 \%$ at 5 years and $87 \%$ at 10 years for the entire group. There was no significant difference in OS among the $3 \mathrm{WHO}$ subtypes (B1 vs B2, $P=.49$; $\mathrm{B} 2$ vs B3, $P=.17$; Figure $1, A)$. There was no significant difference in OS among the 4 Masaoka stages (I vs II, $P=.26$; II vs III, $P=.61$; III vs IVa, $P=.3$; Figure $1, B$ ).

Among the 79 patients who had complete resection, tumor recurrence occurred in $1(5.9 \%)$ with type B1 thymoma, $2(5.7 \%)$ with type B2 thymoma, and $2(7.4 \%)$ with type B3 thymoma. All of these had Masaoka stage III disease (Table 3 ). Of the 5 patients with recurrent disease, 4 patients had pleural dissemination and 1 had pulmonary metastasis. All but 1 of these patients had undergone adjuvant radiation therapy (Table E2). Of the 21 patients who had incomplete resection, $3(14.3 \%)$ patients have died of thymoma during follow-up, whereas $18(85.7 \%)$ were still alive even with progressive disease at the last follow-up point.

DFS at 5 years was $93 \%$ for patients with type B1 thymomas, $85 \%$ for type B2 thymomas, and $82 \%$ for type B 3 thymomas (Figure 2, A). DFS at 5 years was 94\%, 100\%, $61 \%$, and $50 \%$ in patients with Masaoka stage I, II, III, and IV disease, respectively (Figure 2, $B$ ). No difference was found in DFS among the 3 WHO subtypes (B1 vs B2, $P=.79$; $\mathrm{B} 2$ vs $\mathrm{B} 3, P=.6)$. However, when based on the Masaoka staging system, there was a significant difference in DFS between 
TABLE 1. Distribution of Masaoka stage according to WHO subtypes for all patients

\begin{tabular}{|c|c|c|c|c|c|c|}
\hline \multirow[b]{3}{*}{ Stage } & \multicolumn{6}{|c|}{ WHO subtype } \\
\hline & \multicolumn{2}{|c|}{ B1 $(n=22)$} & \multicolumn{2}{|c|}{ B2 $(n=43)$} & \multicolumn{2}{|c|}{$\mathrm{B3}(\mathrm{n}=\mathbf{3 5})$} \\
\hline & No. & $\%$ & No. & $\%$ & No. & $\%$ \\
\hline I & 10 & 45 & 19 & 44 & 9 & 25 \\
\hline II & 5 & 23 & 9 & 21 & 10 & 29 \\
\hline III & 4 & 18 & 8 & 19 & 10 & 29 \\
\hline IVa & 3 & 14 & 7 & 16 & 6 & 17 \\
\hline
\end{tabular}

Masaoka stage II and stage III disease and between Masaoka stage III and stage IVa disease (I vs II, $P=.26$; II vs III, $P$ $=.028$; III vs IVa, $P=.002$; Figure $2, B$ ). No significant factors were found to be associated with overall survival in both univariate and multivariate analyses. With regard to DFS, Masaoka stage (hazard ratio, 3.11; 95\% confidence interval, $1.5-6.46 ; P=.002$ ) and tumor size (hazard ratio, $1.47 ; 95 \%$ confidence interval, $1.16-1.87 ; P=.002$ ) were identified as independent factors that affected survival in multivariate analysis.

\section{DISCUSSION}

The current WHO classification of thymic epithelial tumors is based on the morphology of the epithelial cells as well as the lymphocyte/epithelial cell ratio. ${ }^{1}$ Since the introduction of the WHO classification system in $1999,{ }^{1}$, several authors have evaluated the prognostic relevance of this histologic classification in patients with thymomas. Okumura and colleagues ${ }^{2}$ reviewed 273 patients with thymic tumors and showed that the WHO histologic types reflect the oncologic behavior of thymoma and that the WHO classification may be helpful in clinical practice for the assessment and treatment of patients with thymoma. Additionally, Nakagawa and associates ${ }^{3}$ demonstrated that the WHO histologic classification significantly correlates with clinical stage and can thus predict the prognosis of patients with thymoma.

There is widespread agreement that the prognosis of type $\mathrm{A}$ and $\mathrm{AB}$ thymomas is superior to that of type $\mathrm{B}$ and $\mathrm{C}$ thymomas. ${ }^{2-7}$ This is related to the observation that the proportion of thymoma at an advanced stage gradually increases from type A to C. However, the prognostic relevance among type B thymoma subtypes is still controversial. Some have shown that patients with type B3 thymomas have worse

TABLE 2. Frequency of incomplete resection according to WHO subtypes and Masaoka stages for all patients

\begin{tabular}{|c|c|c|c|}
\hline \multirow[b]{3}{*}{ Overall (\%) } & \multicolumn{3}{|c|}{ WHO subtype } \\
\hline & B1 & B2 & B3 \\
\hline & $5 / 22(23)$ & $8 / 43(19)$ & $8 / 35(23)$ \\
\hline Stage I & $0 / 10$ & $0 / 19$ & $0 / 9$ \\
\hline Stage II & $0 / 5$ & $0 / 9$ & $0 / 10$ \\
\hline Stage III & $2 / 4$ & $3 / 8$ & $2 / 10$ \\
\hline Stage IVa & $3 / 3$ & $5 / 7$ & $6 / 6$ \\
\hline
\end{tabular}



FIGURE 1. A, Comparison of overall survival of all patients among WHO subtypes. B, Comparison of overall survival of all patients among Masaoka stages.

prognosis than those with type B2 thymomas, ${ }^{2,3}$ whereas others have reported no significant difference in survival between these 2 groups. ${ }^{4,5,9,10}$ This discrepancy in the prognostic significance of the WHO classification, especially within type B thymomas, is one of the reasons that our study focused on this specific type of thymomas. In our series, we found no significant differences in the incidence of recurrence and survival among the 3 subtypes of type B thymomas.

These findings could be interpreted in several ways. First, there is substantial interobserver variability in the histologic typing of thymic epithelial tumors. Shimosato ${ }^{11}$ reported that the difference between type B2 and B3 thymomas appears difficult to distinguish and the definition of the 2 subtypes varies among authors. Rieker and associates ${ }^{10}$ assessed the interobserver agreement within various histologic subtypes, based on the WHO classification. The interobserver agreement was good for all WHO histologic types, but it was only moderate for type B tumors. Second, the WHO classification does not clearly specify the definition of combined thymomas, which contain more than one 
TABLE 3. Frequency of tumor recurrence according to WHO subtypes and Masaoka stages for completely resected patients

\begin{tabular}{|c|c|c|c|}
\hline \multirow[b]{3}{*}{ Overall (\%) } & \multicolumn{3}{|c|}{ WHO subtype } \\
\hline & B1 & B2 & B3 \\
\hline & 1/17 (5.9) & 2/35 (5.7) & $2 / 27(7.4)$ \\
\hline Stage I & $0 / 10$ & $0 / 19$ & $0 / 9$ \\
\hline Stage II & $0 / 5$ & $0 / 9$ & $0 / 10$ \\
\hline Stage III & $1 / 2$ & $2 / 5$ & $2 / 8$ \\
\hline Stage IVa & - & $0 / 2$ & - \\
\hline
\end{tabular}

The number of patients with tumor recurrence was counted among patients undergoing complete resection.

WHO histologic subtype. In a report by Chen and associates, ${ }^{4}$ thymomas were classified as type B3 when the B3like areas accounted for more than $30 \%$ of the total area. Conversely, in a study by Nakagawa and coworkers, ${ }^{3}$ thymomas were classified as type B3 when there were any areas of B3 histologic features. However, Ströbel and colleagues ${ }^{5}$ pointed out that combined thymomas share similar clinical findings with their noncombined counterparts with the most aggressive component of the tumor. In our series, although a pathologist reviewed all specimens according to the current WHO classification, the proportion of one subtype to another in combined thymomas was not fully quantified on the basis of established guidelines. Tumors that are predominantly type B1 but contain a small amount of type B3 tumor may not represent "pure" B1 tumors. However, it is difficult to know how much this would have affected the outcome of this study.

The inability of the WHO classification to differentiate prognosis in our series is also related to the fact that there was no significant correlation between WHO histologic types and Masaoka stages in our study population. This is in contrast to previous reports in which the WHO types closely correlated with the Masaoka stages. ${ }^{2-7}$ If we had dealt with the full spectrum of histologic types and had not focused on type B thymomas, the correlation might have been more obvious. However, it should be noted that type B1 tumors at an advanced stage and type B3 tumors at an early stage were relatively more frequent in our series than in previous studies. Although the WHO histologic types reflect the biologic nature or aggressiveness of thymomas, the extent of the disease mainly depends on whether the tumor is diagnosed at an early or advanced stage. This is why the WHO classification failed to show prognostic relevance in our study. Type B3 thymomas, detected at an early stage, could be completely resected despite the biological aggressiveness, and this can be translated into a good prognosis. In the present study, although there was no difference in DFS among WHO subtypes, we found that differences in DFS were significant among Masaoka stages. Therefore, the Masaoka staging system, which reflects the extent or invasiveness of thymomas, can predict the prognosis better
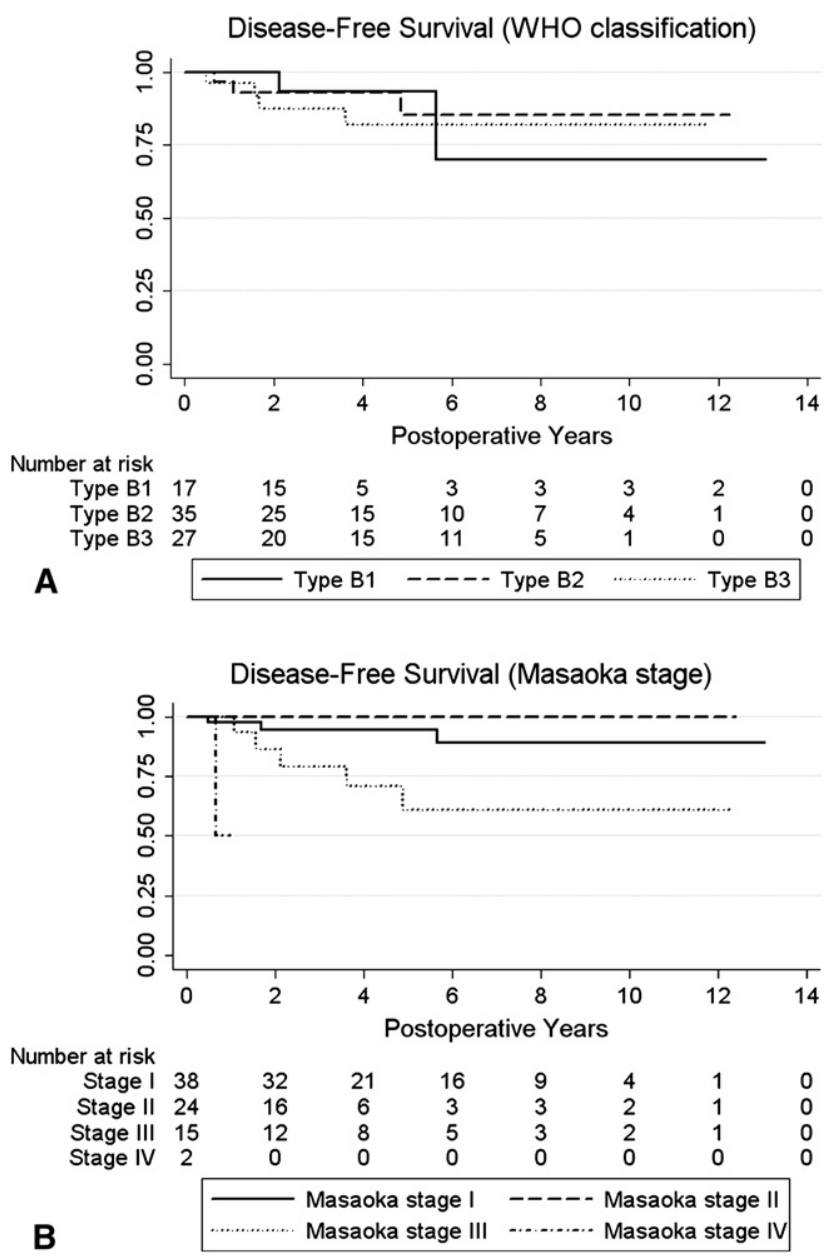

FIGURE 2. A, Comparison of disease-free survival of patients undergoing complete resection among WHO subtypes. B, Comparison of disease-free survival of patients undergoing complete resection among Masaoka stages.

than the WHO classification, and tumor invasiveness or extent is the most important prognostic factor. ${ }^{7,12-14}$

Given the chance of recurrence for type B2 or B3 thymomas at an advanced stage, adjuvant therapy should be considered in such patients. In our study, 5 patients had a recurrence during follow-up after complete resection, and among these, all but 1 patient underwent adjuvant radiotherapy. This suggests that adjuvant radiation therapy was not effective in these patients. Inasmuch as 4 patients had pleural dissemination and 4 had pulmonary metastasis, mediastinal irradiation alone might not prevent pleura-based recurrence. ${ }^{15}$ On the other hand, among the 47 patients who underwent complete resection for type B2 or B3 thymomas at Masaoka stage I or II, $37(79 \%)$ received adjuvant radiation therapy, whereas $10(21 \%)$ did not. During follow-up, none of the patients had a recurrence irrespective of adjuvant therapy. These results raise questions about the need for adjuvant therapy in patients with completely resected stage I or II thymomas, even for those with type B2 or B3 histologic characteristics. Adjuvant radiotherapy has been advocated 
by many authors for completely resected stage II thymomas of the high-risk type B2 or B3 subtype, ${ }^{15-18}$ whereas 2 recent studies dealing with this problem found no significant differences between treated and untreated patients. ${ }^{19,20}$ More prospective data are required to assess the benefit of adjuvant radiation therapy in patients with completely resected stage II type B2 or B3 thymomas.

Because our data were retrospectively collected and analyzed, our study has intrinsic limitations. Although patients with advanced stages of thymomas received adjuvant treatment, indications of adjuvant therapy were not consistent, and this might have affected the outcomes. The study population was rather small, limiting the power of our findings. Furthermore, as described earlier, combined thymomas were not meticulously dealt with in our study. Despite these limitations, however, tumor recurrence was significantly associated with advanced Masaoka stages rather than WHO types, and Masaoka stage was the most important prognostic factor in patients with type B thymoma.

In summary, we retrospectively reviewed the recurrence pattern and long-term survival in patients who underwent thymectomy for WHO type B thymomas. We compared the prognosis among subtypes within type B thymomas according to both Masaoka staging system and the WHO classification system. The long-term results demonstrated that tumor recurrence was significantly associated with advanced Masaoka stages, irrespective of the WHO classification. No significant difference was found in DFS among the histologic subtypes, whereas there were significant differences in DFS among the Masaoka stages. Given the heterogeneity of WHO type B thymomas, Masaoka stage should always be considered when predicting prognosis and planning treatment, including adjuvant treatment for patients with type B thymomas. Our results should be confirmed by other prospective studies.

\footnotetext{
References

1. Rosai J, Sobin LH. Histological typing of tumors of the thymus. World Health Organization International Histological Classification of Tumors. 2nd ed. New York (NY): Springer-Verlag; 1999.

2. Okumura M, Ohta M, Tateyama H, Nakagawa K, Matsumura A, Maeda H, et al. The World Health Organization histologic classification system reflects the onco-
}

logic behavior of thymoma. A clinical study of 273 patients. Cancer. 2002;94 624-32.

3. Nakagawa K, Asamura H, Matsuno Y, Suzuki K, Kondo H, Maeshima A, et al Thymoma: a clinicopathologic study based on the new World Health Organization classification. J Thorac Cardiovasc Surg. 2003;126:1134-40.

4. Chen G, Marx A, Wen-Hu C, Yong J, Puppe B, Stroebel P, et al. New WHO histologic classification predicts prognosis of thymic epithelial tumors. A clinicopathologic study of 200 thymoma cases from China. Cancer. 2002;95:420-9.

5. Ströbel P, Bauer A, Puppe B, Kraushaar T, Krein A, Toyka K, et al. Tumor recurrence and survival in patients treated for thymomas and thymic squamous cell carcinomas: a retrospective analysis. J Clin Oncol. 2004;22:1501-9.

6. Kondo K, Yoshizawa K, Tsuyuguchi M, Kimura S, Sumitomo M, Morita J, et al. WHO histologic classification is a prognostic indicator in thymoma. Ann Thorac Surg. 2004;77:1183-8.

7. Kim DJ, Yang WI, Choi SS, Kim KD, Chung KY. Prognostic and clinical relevance of the World Health Organization schema for the classification of thymic epithelial tumors. A clinicopathologic study of 108 patients and literature review. Chest. 2005;127:755-61.

8. Masaoka A, Monden Y, Nakahara K, Tanioka T. Follow-up study of thymomas with special reference to their clinical stages. Cancer. 1981;48:2485-92.

9. Chalabreysse L, Roy P, Cordier JF, Loire R, Gamondes JP, Thivolet-Bejui F. Correlation of the WHO schema for the classification of thymic epithelial neoplasm with prognosis: a retrospective study of 90 tumors. Am J Surg Pathol. 2002;26: 1605-11.

10. Rieker RJ, Hoegel J, Morresi-Hauf A, Hofmann WJ, Blaeker H, Penzel R, et al Histologic classification of thymic epithelial tumors: comparison of established classification schemes. Int J Cancer. 2002;98:900-6.

11. Shimosato Y. Controversies surrounding the subclassification of thymoma Cancer. 1994;74:542-4.

12. Dawson A, Ibrahim NB, Gibbs AR. Observer variation in the histopathological classification of thymoma: correlation with prognosis. J Clin Pathol. 1994;47: 519-23.

13. Quintanilla-Martinez L, Wilkins EW Jr, Choi N, Efird J, Hug E, Harris NL. Thymoma. Histologic subclassification is an independent prognostic factor. Cancer. 1994;74:606-17

14. Suster S, Moran CA. Thymoma, atypical thymoma, and thymic carcinoma. A novel conceptual approach to the classification of thymic epithelial neoplasma. Am J Clin Pathol. 1999;111:826-33.

15. Ogawa K, Uno T, Toita T, Onishi H, Yoshida H, Kakinohana Y, et al. Postoperative radiotherapy for patients with completely resected thymoma. A multi-institutional, retrospective review of 103 patients. Cancer. 2002;94:1405-13.

16. Schmidt-Wolf IG, Rockstroh JK, Schüller H, Hirner A, Grohe C, MüllerHermelink HK, et al. Malignant thymoma: current status of classification and multimodality treatment. Ann Hematol. 2003;82:69-76.

17. Gripp S, Hilgers K, Wurm R, Schmitt G. Thymoma: prognostic factors and treatment outcomes. Cancer. 1998;83:1495-503.

18. Eralp Y, Aydiner A, Kizir A, Kaytan E, Oral EN, Topuz E. Resectable thymoma: treatment outcome and prognostic factors in the late adolescent and adult age group. Cancer Invest. 2003;21:737-43

19. Singhal S, Shrager JB, Rosenthal DI, LiVolsi VA, Kaiser LR. Comparison of stages I-II thymoma treated by complete resection with or without adjuvant radiation. Ann Thorac Surg. 2003;76:1635-42.

20. Venuta F, Rendina EA, Longo F, Giacomo TD, Anile M, Mercadante E, et al Long-term outcome after multimodality treatment for stage III thymic tumors. Ann Thorac Surg. 2003;76:1866-72. 
TABLE E1. Treatment modalities according to WHO subtypes for all patients

\begin{tabular}{|c|c|c|c|c|c|c|}
\hline & \multicolumn{6}{|c|}{ WHO subtype } \\
\hline & \multicolumn{2}{|c|}{ B1 $(n=22)$} & \multicolumn{2}{|c|}{ B2 $(n=43)$} & \multicolumn{2}{|c|}{ B3 $(n=35)$} \\
\hline & No. & $\%$ & No. & $\%$ & No. & $\%$ \\
\hline Complete resection & 17 & 77 & 35 & 81 & 27 & 77 \\
\hline \multicolumn{7}{|l|}{ Treatment } \\
\hline Surgery alone & 9 & 41 & 6 & 14 & 11 & 31 \\
\hline Surgery + RT & 12 & 54 & 33 & 77 & 22 & 63 \\
\hline Surgery $+\mathrm{CT}$ & - & - & 4 & 9 & 1 & 3 \\
\hline Surgery + CRT & 1 & 5 & - & - & 1 & 3 \\
\hline
\end{tabular}

$R T$, Radiation therapy; $C T$, chemotherapy; $C R T$, chemoradiation.
TABLE E2. Frequency of tumor recurrence according to WHO subtypes, Masaoka stages, and radiation therapy for patients undergoing complete resection

\begin{tabular}{|c|c|c|c|c|c|c|}
\hline & \multicolumn{6}{|c|}{ WHO subtype } \\
\hline & \multicolumn{2}{|c|}{ B1 } & \multicolumn{2}{|c|}{ B2 } & \multicolumn{2}{|c|}{ B3 } \\
\hline & RT & No RT & RT & No RT & RT & No RT \\
\hline Stage I & $0 / 5$ & $0 / 5$ & $0 / 16$ & $0 / 3$ & $0 / 6$ & $0 / 3$ \\
\hline Stage II & $0 / 4$ & $0 / 1$ & $0 / 8$ & $0 / 1$ & $0 / 7$ & $0 / 3$ \\
\hline Stage III & $1 / 2$ & - & $2 / 4$ & $0 / 1$ & $1 / 7$ & $1 / 1$ \\
\hline Stage IVa & - & - & - & $0 / 2$ & - & - \\
\hline Stage I & $0 / 5$ & $0 / 5$ & $0 / 16$ & $0 / 3$ & $0 / 6$ & $0 / 3$ \\
\hline
\end{tabular}

$R T$, Radiation therapy. 